\title{
GLANDULAS SUPRARRENAES
}

\author{
DR. D. M. GONZALEZ TORRES
}

\section{ANATOMIA}

Situadas acima do pólo superior do rim e aplicadas contra a parede posterior abdominal pelo peritôneo, têm a forma de uma meia lua, e pesam de 10 a 11 grs. no adulto.

Nelas se descrevem duas partes apesar de que seus limites não sejam nitidos pois que ambas se unem: a cortical e a medular, e a esta dualidade anátomica corresponde tambem uma dualidade funcional e de origem.

A cortical - E' formada por células sustentadas por uma fraca rede de tecido conjuntivo vascular proveniente da cápsula que rodeia o órgão.

As células estão dispostas em tres zonas indo da superficie para a profundidade: a zona externa de células redondas, a média ou fasciculada de células em cordões, a interna ou reticular de células em ređe com granulações pigmentárias.

Estas células da cortical contem grânulos lipoides de lecitina e cefalina.

A Medular - E' composta por células dispostas irregularmente em rede, em meio de um tecido conjuntivo de sustentação, muito vascularizado. São células cromafinicas, assim chamadas porque tratadas pelo ácido crômico ou cromatos, coloram-se em amarelo-escuro (com o cloreto de ferro coloram-se en verde). São células poligonais, de 15 a 35 micras, e de protoplasma basófilo.

A cortical deriva do mesoderma (epitélio celomático), de um bróto formado perto do corpo de WoLFF, isto é, tem a mesma origem que o aparelho urogenital. Mais tarde, agregam-se células cromafinicas e fibras simpaticas que formarão a medular. As células da medular derivam das simpatogonias (derivado ectodérmico) que além da série cromafinica, é origem da série simpatica.

Existem em outras regóes além da renal, formações suprarrenais aberrantes e do mesmo significado:

(1) Capitulo VI da Parte Especial do livro "Endocrinologia" do Prof. Gonzales Torres editado nestes dias. 
Formasōes Córtico-Suprarrimuis: Din tecido celular retroperit'meal regiâo do pancreas e figado, genital. etc.

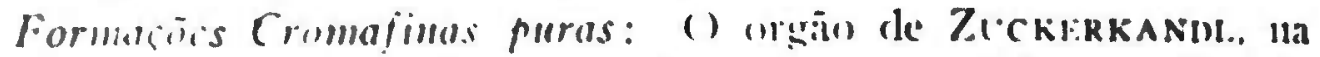
origem da mesentérica inferior. "glomus retrocarotideo, a glàndula coxigea de I.uschka, no tecirlo adiposo da ponta do coccis, etc..

A innprrtancia funcional da glandula suprarrenal ć grande $\mathrm{c}$ conlecida ha nuuito tenıpo. Porém, sómente, nos últimos anos, é que ficou conheciclo mellor o papel que corresponde a cada una de suas partes. Com os progressos da técnica e da fisiologia podem ser feitas extirpaçóes parciai., injetar extratos. tambem parciais, e cutuclar experimentalmente certos quadros que destle Ampison, nos meiarlos do séculu passado, chamavam a at'nç̧ão sem porler explicar-se claranlente o papel que cahia á medular c'á cortical.

Aprear de us noswos conhecinentos atuais nos permitir conhecer a papel da medular e da cortical, devemos notar que ambas as partes,

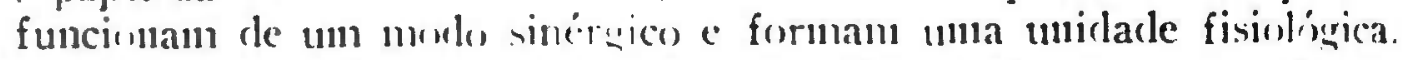

1 - A extirpação las suprarrenais é mortal $\mathrm{cm}$ quasi todos os casus e en todos us aninlais. Casos de solorevivencia, mais ou menos 1::olongada devem ser atribuidas ás formaçōes aberrantes descriptas, ¿ que se apresentam no fin de um certo tempo, hipertrofiados. A extirpação de uma delas geralmente não causa a morte e si sacrificarmos o animal, um tempo depois observaremos uma hipertrofia notavel da que ficou intacta.

2 - Os aninais privalus da suprarrenal apresentam um quadro clinico caracterizado por: queda rápirla da pressiós global, astenia, e adinamia intensa e progressiva. rápida fadiga muscular, grande emagrecimento, sonolência, $e$ um quadro humoral caracterizado por hipoglicemia, hipocloremia, diminuição da reserva alcalina, aumento do potássio. colesterina, urea, fosfatos, maior sensibilidade para a insulina.

3 - O animal oferece rrancle diminuicão de resistencia ás infecções em geral e aos tóxicos.

\section{OS HORMONIOS}

A medular - Suas células produzem a adrenalina ou epincfrina ou suprarrenina, sendo tambem produzida pelas células cromafinicas. Isolada e crystalizada em 1901 por Takamine e Alrich, Stolz em 1904. é um pó bránco soluvel em ácidos diluidos, muito pouco solúvel na água $(1: 10.000)$ e no álcool, insolúvel em toluol, henzina. clorofórmio. e correspondente á formula :

$$
\begin{array}{lllll}
\mathrm{C}_{9} & \mathrm{H}_{13} & \mathrm{~N} & \mathrm{O}_{3}
\end{array}
$$

Seu ponto de fusão é de $263^{\circ}$. E' toxica em doses elevadas ( 5 mgrs. por quilograma de peso para o cachorro) por via que não 
seja bucal, e produz necroses do tecido celular do lugar onde for injetada.

Por dosagens feitas em cadáveres por G. BAYER, acharam-se quantidades variaveis segundo a idade, entre 0,20 e 0,40 mgrs. de adrenalina para cada grama de suprarrenal.

Sua sintese se faz por varios métodos obtendo-se uma forma racêmica e no comércio usa-se uma forma que contem um pouco da dextrogira.

Propiedades: E' um excitador đo simpático. As injeções endovenosas ou intramusculares, produzem vaso constriç̧ão capilar e arterial e por esta ação periférica, elevam a pressão. As contracçôes cardiacas aumentam de força e de amplitude; taquicardia.

Atua sobre os músculos lisos, contrae os esfinteres, (cardiaspiloro ileo cecal, anal) (*). Relaxa os músculos lisos da traquéa e brônquios; age sôbre (paraliza) o intestino, vias biliares. Contrae o utero em forma continua - Dilatação papilar e exoftalmus. Sua ação se produz ao nivel das terminações simpáticas da placa mioneural. Excita a contração dos músculos lisos. Mobilisa as reservas de glicogeno hepático, eleva a glicemia; e produz, ás vêses glicosúria segundo a dose em que se emprega.

Atua sobre o centro termoregulador, produzindo aumento da temperatura. Estimula as combustões organicas. Aumenta o metabolismo celular e o consumo de $\mathrm{O}^{2}$.

A cortical - A cortical suprarrenal é rica em lipoides, e dela tem-se isolado: lecitina, colesterina, fosfatides e fosfatase, caroteno (substancia base da vitamina A.), vitamina C. (é o órgão mais rico em vitaminas C.) ; colina, considerada por MAGNuS, o hormonio dos movimentos intestinais; lipides que se coram com o acido osmico e são soluveis no cloroformio, eter, e alcool; estes lipides formam o $35-50 \%$ do extrato total cortical. Tambem glutation.

$\mathrm{Da}$ cortical foram isolados os seguintes Hormonios:

1 - A cortina de Hartmann considerado o hormonio cortical por excelencia, cuja formula é:

\section{$\mathrm{C} 20 \mathrm{H} 30 \mathrm{O} 5$}

Injetado em fortes doses não produz intoxicação. Além disso, parece ter um antagonismo com a tiroxina. Pode ser cristalizada, é pouco soluvel em agua, um pouco mais no eter e acetona. E' bastante resistente á ebulição, fermentos proteoliticos e acidos diluidos. Estimula o crescimento e tem acção morfogenetica; a hiperfuncção cortical nos meninos causa um atletismo e hirsutismo precoce, e macrosomia precoce, e nos adultos crescimento exagerado, hirsutismo e obesidade. Aumenta a resistencia á fadiga, excita a sintese intramuscular do acido lactico, produzido depois da fadiga.

(*) Uma dose excessiva produz ileus paralitico $\mathrm{ccm}$ contração forte dos esfíncteres. 
Corrige a aç̧ão da extirpação do cortex. A extirpaçấo do cortex int sta insuficiencia causa astenia, fadigabilidade nuscular. anorexia. hipocloridria. furte deshidratação, pigmentação hrumzecada dia" pele c das mucosas. Tem açaáo sobre os caracteres sexuaes secunharios ha uma masculinizacão fisica e psiquica das mulheres com hiperplasia ou tumor da suprarrenal. Intervem no metabolismo dow slucidos, por mecanismo em sentido inverso da adrenalina: aumenta a glicemia e o glicogenio hepatico e muscular: aumenta tambem o glutation, e liminuc a urea. A extirpação do cortex ou sua insuficiencia produz hipoglicemia e maior sonsibilidade $a$ insulina, e diminuiçāo do colesterol sanguineo. Regulariza o metabolismo mineral. A extirpação do cortex ou a insuficiencia aumenta a eliminação urinaria do $\mathrm{Cl}$. Na. c diminue sua concentração no sangue, - aumenta a protasemia. A cortina corrige esses efeitos. Aumenta a resistencia ás infecçōes $\mathrm{c}$ toxicos e corrige as susceptibilidades ás reaçōes anafilaticas que produz a insuficiencia ou extirpação do cortex.

2 - A Corticostirona do Reichstein (1937) e Steigert, tem por formula:

\section{C21 H30 O4,}

e é necessária á vida. Mantem vivos os animais descorticados. Não foi preparada por sintese. Porem, os mesmos autores obtiveram por sintese partindo do estigmasterol, um composto vizinho, a desoxicorficosterona (cortenil) que tem um grupo $\mathrm{OH}$ menos que a corticosterona, e com formula muito parecida a da testosterona e progesterona, a vitamina $D$, e us acillıs biliares. A ação desta hormona sintetica em ratos surrenalectomizados é mais forte que a da corticosterona e tem as mesmas propiedades que os melhores extratos glandulares ou a Cortina, considerando-se, portanto a verdadeira hormona cortical, $1 \mathrm{mgr}$. de desoxi-corticosterona em solução oleosa corresponde a $3 \mathrm{cc}$. de um estrato activo da Cortina, e o seu preço é menor.

3 - A Adrenosterona de Reichstein (1936) de formula:

\section{C19 H24 O3}

atua sobre a formação e desenvolvimento dos caracteres sexuais masculinos secundarios. Sua deficiencia produz no homem uma diminuição da potencia e da libido; enjetada numa mulher normal proluz um encurtamento dos intervalos menstruaes.

\section{RELAÇOES COM OUTRAS GLANDULAS}

Com a hipofise- a cortical: No capitulo, destinado á hipofise, nos ocupamos do hormónio hipofisário corticotropo ou corticoestimulina. Ali diziamos que a hipofisectomia determinava atrofia e degeneração do cortex e que a injeção consecutiva de extrato hipofisário anulava o efeito da hipofisectomia. 
A medular: Injetando juntos: extrato de lóbulo posterior e adrenalina, esta não produz seu efeito. Disso, deduz-se a existencia de certo antagonismo entre ambas as substancias. A pituitrina aumenta o peristaltismo e relaxa os esfinteres.

Com a tiroide: Nos casos de hipertiroidismo observa-se uma diminuição dos lipoides corticais. A tiroidectomia os aumenta. A injeção de extrato cortical aumenta o iodo tireoideo. Das experiencias de vários autores se deduz haver um antagonismo entre a tireoide e a cortical quanto á regulação térmica. A secreção tiroidea sensibiliza as terminações simpáticas para a acção da ađ̛renalina, e a injeção de extrato tiroideo aumenta a secreção adrenalinica.

Com o Timo: A Timectomia produz hiperplasia da medular; e a extirpação da surprarrenal retarda a involução do timo.

Com as glandulas sexuais: Os animais de grande poder de reprodução possuem volumosas suprarrenais e vice-versa (G. BAYER).

A cortiçal tem ação masculizante e excita a maduração sexual; durante a gravidêz se observa hiperplasia da cortical. A castração ảetermina hipertrofia das suprarrenais e a ablação destas produz atrofia genital.

As relações com a medular são menos claras; por fim parece haver antagoǹismo entre Hormona cortical e adrenalina.

\section{TESTES}

1 - Dosagem da colesterina: Normal: $1,30-1,70$ grs. $\%$ oo de sangue; aumentada na hiperfunção.

Do potássio:

Clorureto de potássio: 0,26. g. \% $\%$.

Sulfato: $0,281 \mathrm{~g} . \%$. 00 .

Potássio: $16-21 \mathrm{mg}$. \% no sôro.

Auméntada na moléstia de Addison.

Do cloro:

Sangue: $450-500 \mathrm{mg} . \%$ em $\mathrm{Cl} \mathrm{Na}$.

Plasmático: $8,3 \mathrm{~g}$. $\%$ oo.

Globular: $1,7-1,9$ g. $\%$.

Diminuido na moléstia de ADDIson e na insuficiencia.

Do sódio:

No sangue total: $1,9 \mathrm{~g} \cdot \%$ oo.

No sôro: $3,2 \%$ g.

A taxa de sódio acha-se diminuida na insuficiencia.

O quociente $\frac{\mathrm{Na}}{\mathrm{K} \times 10}$ é superior à 1,5 (Annes DIAS); segundo 
o mestre brasileiro na insuficiencia suprarrenal este quociente desce de acirdo com a gravidade. e cifras menores de 1 indicam geralmente casns sérios.

2 - Para o simpático:

prova da glicosúria alimenticia;

prova da glicosuria adrenalinica:

reflexo óculo cardiaco.

3 - Metabolismo basal: diminuido 20-30\% no AdDison.

4 - Prova do ergngrafo (Porak e Stevenis) que demonstrará a adlinamia $e$ a ripicla fadigabilidarle muscular do Addison.

5 - Prova da climinação do cloreto sodicn, de Powrer e Wurner.

Deixar o doente $\mathrm{em}$ dieta pobre $\mathrm{cm}$ cloreto de sodio, e rica em potassio durante dois dias e mein. No individuo normal, se produz I'ma diminuição da climinação desses elementos pela urina: na insuficiencia suprarrenal ha um aumento constante da eliminação urinaria do cloreto de sodio.

Técnica: Dar ao doente durante 2 dias e meio dieta com:

$$
1,7 \text { grs. de cloreto de sodio diario; }
$$

4,3 grs. de potássio.

No $2 .^{\circ}$ dia, dar-lhe 40 .cc. de liquido por kilo de peso corporal.

Recolher a urina: no $1 .^{\circ}$ e $2 .^{\circ}$ lia, ás 8 da manhã e ás 8 da noite; $3 .^{\circ}$ dia, ao meio dia.

Recolher o sangue: no $2 .^{\circ}$ dia, ás 8 da manhã e $3 .^{\circ}$ dia, ás 10 da nanlıã.

Resultado: Concentração urinaria do cloreto de sodio na manhã (i) $3 .^{\circ}$ dia, igual a $125 \mathrm{mg} . \%$ na função normal da suprarrenal.

Nas insuficiencias: $225 \mathrm{mg} . \%$.

As cifras intermedias não são concluentes, e a prova deve ser extendicla a 6 dias.

6 - Prova da insulina da Marañon:

Dosar a glicemia em jejum;

Injetar intramuscularmente, $10 \mathrm{U}$. de insulina.

A prova é positiva quando a glicemia baixa de $30 \%$ da taxa inicial. los $40 \%$ dos casos positivos: trata-se de insuficiência suprarrenal.

\section{PATOLOGIA CLINICA}

Hipof unção:

Hipofunção Global Suprarrenal.

Sindrome de Addison.

Forma aguda de insuficiência.

Formas frustres. 
Hiperfunção:

Medular: sindroma de hipersurrenalismo.

Cortical: sindroma genitosuprarrenal: pseudo-hermafrodismo.

Pubertas precox.

Virilismo e hirsutismo.

Formas frustres.

\section{HIPOFUNCC̨ÃO GLOBAL SUPRARRENAL}

\section{Etiologia :}

Infecções agudas: grippe, tifo, pneumonia, paludismo, etc. ou cronicas: tuberculose, sinusites, amigdalites, difteria, toxemias.

\section{Sinais Clinicos:}

astenia, cansaço facil sem vontade de nada;

anemia hipocromica;

anorexia, prisão de ventre;

dores vagas no hipogastrio e região lombar;

transtornos menstruaes; diminuição da libido e da potencia, hipotensão, pulso depresivel ;

facilidade para apanhar infecções, pela diminuição das defesas;

M. Basal: ligeiramente diminuido (15-20\%);

acção dinamico especifica proteica baixa;

hipoglicemia e aumento da tolerancia aos $\mathrm{H}$. de $\mathrm{C}$.

\section{Tratamento:}

Regime rico em vitamina $\mathrm{C}, \mathrm{H}$. de $\mathrm{C}$. cloretos, albuminas, pouca gorduras;

Extrato suprarrenal total. Cortina, 1-2cc. diarios;

Extrato hipofisario e tiroideo;

Tonicos em geral, e sobretudo estricnina.

\section{SINDROMA DE ADDISON (1855)}

Evolução: Começo insidioso com emagrecimento, dores lombares, evolução progressiva e lenta que pode durar anos seguidos, ás: vezes havendo crises agudas e insuficiencia, ou remissões. Quasi sempre letal.

Etiologia: Segundo Marañon (5), 86\% dos addissonianos pertencia á constituição astênicas ou hipoplasica. E' frequente $o$ estado timolinfático.

As lesões observadas são de tuberculose, no $80 \%$ dos casos mais ou menos e quasi sempre da forma caseosa. 
Simptomas: GRFine, RowntreE e outrus contam 3 periolos no decurso da evolução da molestia de Addisson: $10^{\circ}$ o periodo de latencia, de destruiçäio glandular e de esgotamento das reservas funcimais: $2 .^{\circ}$ periodo clinico ou de insuficiencia parcial; $3 .^{\circ}$ periodo tcrminal, de insuficiencia total, periodos das crises.

Astenia: Adinamia, esgotamento muscular rápido, verifica-se pelo ergógrafo, atrofia muscular, grande sensação de fadiga, reação elétrica normal. A lassitude do começo transforma-se mais tarde em apatia completa, com conservação da inteligencia.

Melanodermia: Pigmentação morena da pele e mucosas no começo parcelada, em pequenas manchas obscuras, depois confluentes; 1:1) rosto, como mascara da gravidêz. Depois, as partes descobertas ou naturalmente pigmentadas tais como: escroto, mamelão, etc. ; mais tarde atinge tambem, as partes cobertas, extendendo-se por todo 0 corpo. São poupados a palma da mão e planta dos pés.

Nas mucosas: conjuntiva ocular, mucosa bucal, aboubada palatina, etc.

Hipotensão: Da naxima e da minima, pequeno indice oscilométrico.

Tendencia á sincope. Importante é a hipotensão ortostatica.

Pulso depressivo, taquicardia.

Criestesia. Hipotermia.

Emagrecincuto: Anemia.

Dores epigástricas ou lonulures. As veses crises solar.

Perturbacōes digestivas: Anorexia progressiva e global. No começo constipação e, após diarréa. Hipocloridria.

Amenorréa ou impotencia.

Linha branca de SERGE.vT: que aparece um pouco tárdiamente (30-60 segundos).

Reflexos normais.

Radiografia: Frequentemente calcificaçōes das suprarrenais.

Metabolismo: Metabolismo basal diminuido entre 20 e $30 \%$. Acçāo dinamico especifica proteica-diminuida.

Cloropenia, hipocloruria; aumento de creatinuria.

Hiponatremia- Hiperpotassemia- hipocólesterinemia.

Frequente hipoglicemia, grande tolerancia aos hidratos de carbono.

Grande sensibilidade á insulina.

Lréa ligeiramente aumentada. Hipocalcemia inconstante.

Diminuição do glutation (6-8-15 $\mathrm{mg}$. de glutation reduzido para cada 100 c.c. de sanguc; Normal: $17-26 \mathrm{mg}$. glutation reduzido /c de sôro; Diminuição ligeira 12-17; marcada 10-12 mg. \%).

Marañox (5) dá suma importáncia ao diagnóstico da fase preaddissoniana da insuficiencia suprarrenal, por ser a única fase em que pode curar-se a molestia. O A. tem em conta a constituição astênica, e os pequenos sintomas como a astenia, anorexia, soluços, pigmentação fácil da pele pelo sol, amenorréas, frequência de abortos, dificuldade de aleitamento, todos êstes sintomas cedem fácilmente pelo uso da cortical suprarrenal e o regime rico em cloreto 
de sódio. No periodo de estado, êle agrupa os sintomas do seguinte modo:

Cortical:

pigmentação ;

emagrecimento ;

anorexia, perturbações digestivas.

Medular :

perturbações circulatórias;

hipotensão ;

perturbações do metabolismo dos $\mathrm{H}$. de $\mathrm{C}$; hipoglicemia.

No periodo terminal:

perturbações do metabolismo, deshidratação;

hipocolesterinemia de prognóstico grave;

hipoglicemia ;

aumento de creatinemia e creatinúria;

aumento do ácido lático no sangue e na urina;

acidose ;

diminuição do $\mathrm{Na}$ e $\mathrm{Cl}$;

aumento do potássio.

Annes Dias, que se tem ocupado muito do assunto, diz que se deve suspeitar da insuficiencia suprarrenal em toda doença infecciosa cujo decurso ou convalescença se acompanhar de grande astenia, hipotensão, crises dolorosas abdominais, etc., sobretudo si ha uma desproporção entre a intensidade destes' fenómenos e a gravidade ou duração da infecção (6).

Anatomia patológica: Como já dissemos, mais ou menos em $80 \%$ dos Addissonianos encontram-se lesões tuberculosas na sua maioria de forma caseosa. Com menor frequencia: sifilis, esclerose ou hipoplasia da cortical. As veses hipoplasia do tecido cromafino.

Diagnóstico: Em certas ocasiões é dificil o diagnóstico diferencial, sobretudo da melanodermia com outros sindromas ou sintomas.

$A$ pitiriase: Ataca os enfermos miseraveis e hiponutridos.

A caquexia palúdica: Os antecedentes, a hepatomegalia.

A cirrose pigmentaria: A hepatomegalia, glicosúria, etc.

Tratamento: A injeção de Cortina de Kendal ou extrato cortical, de Wirson ou extrato suprarrenal total dá excelentes resultados. O Hormonio sintético: disoxy-corticosterona de ReICHSTEIN e Von Euw, subcutaneo, nas dóses diarias de 5 a $25 \mathrm{mgr}$. ou bem pelo metodo de ThORN-Engel e EISENBERG de implantar sob a pele, e com anestesia local, um comprimido de $200 \mathrm{mg}$. desse hormonio; a desintegração lenta forma um hormonio durante meses.

$1 \mathrm{mg}$. de acetato de desoxicorticosterona corresponde a 10 c.c. de Cortina.

Agregar vitamina C. (Até uma gr. diario endovenoso). 
Eschatin Parke Davis- frascos de 10 e $50 \mathrm{cc} .1 \mathrm{cc} .=40 \mathrm{gr}$. orgão $=10 \mathrm{~L}^{\circ}$ cachorro.

Extrato suprarrenal total: 0,20- 1 gr. diáriamente.

Adrenalina sol. a $/ \mathrm{on}^{-10} \mathrm{mg}$. por dia "per os", ou intramuscularmente $1 \mathrm{mg}$.

Nas formas crônicas- $0,10-0,20 \mathrm{gr}$. de extrato diáriamente.

RIvorRe aconselha :

0,20 ur. cloriclrato de cisteina em 2 c.c. de agua (esterilisada 2 veses a $60^{\circ}$ ) intravenosamente, cada 2 dias, misturando-se a 20 c.c. die sol. salina a $10^{\text {? }}$, no momento do uso. Ou bem: 0,10 gr: clorydrato cisteina endovenoso diáriamente ou em dias alternados.

Estricnina, nux vomica.

Regime: Hillratos de carbono $\mathrm{em}$ grandes quantidades. Pouca gordura.

Regime salgado:

bacalhau, azeitonas, carne salgada etc.

Citratu de sóllio . ... ........ 5 gr.

Clorureto de sódio .............. 10 "

$\mathrm{P} / 1$ papel diário em água.

Em casos graves, injetar gota a gota endovenosamente:

Citrato cle sódio $\ldots \ldots \ldots \ldots \ldots \ldots .0,5 \mathrm{gr}$.

Clorureto de sódio .............. 1 "

Glicose .................... 10 "

Agua ..................... 100 cc.

Até 3-4 litros em um dia.

Pouco potássio. Isto consegue-se fervendo 2 a 3 veses os legutmes. renovando a água (são ricos em potássio: carne, peixe, couveflor: ervilha, batatas, espinafre, frutas secas). São pobres em potássio: trigo, abobora, espargo, cenoura, oliva.

$E^{\prime}$ indicado o uso do vinho tinto seco com carne vermelha $e$ vinho seco branco com carne branca, sobretudo nos casos de inapeténcia, limitando-se a quantidade de água nas refeições. Aconselha-se tambem. em casos de anorexia, tomar um hora antes do almoço e 4) jantar 100-120 grs. de glicose em água, o que tambem representa um grande valor calórico.

Tas crises graves de insuficiencia (vomitos- diarréa- forte dôr (pigástrica, colapso. etc.), usa-se na clinica de Mayo:

Repouso absoluto, não palpar abdomen.

Retirar sangue para as dosagens de L'réa- Glicose- Cloretos e iijetar endovenoso sol- $10 \%$ glicose com $1 \% \mathrm{Cl} \mathrm{Na}$ e $0,5 \%$ citrato de sódio e 30 c.c. de extrato cortical. Depois de 1 hora o quadro melhora: seguir injetando até que em 12 horas o doente receba 3-4 litros e $50 \mathrm{cc}$. de extrato cortical. 
No dia seguinte dar baśtante liquido ao doente e 1 litro diario de Elixir de Addison: Sol. acuosa contendo $10 \%$ de $\mathrm{Cl} \mathrm{Na}$ e $5 \%$ de citrato com extratos de frutas. Injetar tambem diáriamente até desaparecer os fenómenos agudos: 2 litros de sol. acima mencionada e $20 \mathrm{cc}$. de extrato cortical.

Thompson e colaboradores (14) preconizam nas crises (nauseas, vomitos, por vezes diarreia, acentuada astenia):

1) Endovenoso $10 \mathrm{cc}$. de extr. cortical (não ha perigo de superdosagem).

sol. dextrosa a $50 \%$ em sol. salina fisiol. a $9 \%=1$ litro $\mathrm{c} / 6$ hs.

para cada 24 hs. pode-se ajuntar a 1 litro deste liquido:

50 cc. de sol. de citrato de sódio a $25 \%$.

Quando o doente melhora:

extrato cortical pelo menos $10 \mathrm{cc}$. diarios.

per os: 12 gr. $\mathrm{Cl} \mathrm{Na}$ e 4 gr. citrato de sódio, diarios.

dieta com valor calorico elevado, porém, pobre em $\mathrm{K}$.

Alguns produtos opoterápicos, sinteticos, etc...

Extrato suprarrenal L. P. B.: comprimidos, injetavel, glicerinado

Iliren Bayer- extrato cortical sem adrenalina. 1 pilula $=$ $=3$ ǵrs glandula fresca; $2-6$ diarios.

Cortin Roche: amp. de $10 \mathrm{cc}$; 1 cc.: 50 grs. glandula fresca.

Eschatin Parke Davis: amp. de 10-50 cc.; 1 cc.: 40 gr. Glandula fresca: $10 \mathrm{U}$. cachorro.

Cortigen Richer: extrato cortical sem adrenalina; $1 \mathrm{amp}=15$ gr. gland. fresca: 1-2 ao dia.

Adrenalina injetavel, per os, para inhalar, etc.

Ephedrina (sudedáneo da adrenalina) alcaloides da Ephedra.

Efetonina (clorhydrato de Efedrina racemica) menos ativa que Efedrina Off.

Cortidyn "Promonta" $-1 \mathrm{cc} .=5$ U. R. cotico- dinamicas.

\section{INSUFICIENCIA AGUDA, OU SUPRARRENALITE \\ HEMORRÁGICA AGUDA, OU SINDROMA DE WATERHOUSE-FRIDERICHSEN}

Etiologia: No decurso de enfermidades infecciosas e geralmente causada por uma hemorragia ou uma trombose.

Evolução: Rápida e grave.

Simptomas: Geralmente, durante uma enfermidade aguda, produz-se o quadro tipico da insuficiência suprarrenal, sem prodromos, bruscamente e em forma grave, com evolução rápida: 
Astenia acentuada, hipotensão, lipoternia, fortes dores lombares uu abdominais com vómitos, que podem simular um quadro aguilo de perturbação; cianose das extremidades, pulso fraco (• irre. gular, taquicardia; mais tárdiamente: purpura gencralizada: uremia ligeira, hipoglicemia.

Anatomia patológica: Henorragia difusa bilateral ou pequenas sufusōes hemorrágicas.

\section{FORMAS FRUSTRAS DO SINDROMA DE ADDISON}

Ou furmas nāo addisonianas da insuficiência suprarrenal, tan1lem chamarlas formas amelanodérmicas do sindroma de Mlilism. ate.

Sãu quadros de insuficiència suprarrenal, sem melanodérmia, furén con outros sintomas:

Astenia. farligr. cmaurcecinento. Mctabolismo:- 10 á -25. Depressão, menopausa precoce, diminuição da libido, transtornos digestwos; dispepsia hijuclórica, anurexia, crises de diarreia, a que se arescenta o quadro sanguineo já descrito.

Hipotensão global, de pequeno indice oscilonétrico. Hijotermia.

ipresenta-se geralmente em tipos hipoplasticos, astênicos e atónicos, gracis. São predispostas as pessôas que fácil e fortemente. se pigmentan com os banhos de sol.

O tratamento deve ser como já indicamos mais acima:

Regime pobre em potássio e em gorduras, rico em $\mathrm{H}$. de C. e em cloreto de sódio, águas cloruradas.

Cirtin. Pancortex, extrato suprarrenal total.

litamina C. Frutas do gênero Citrus (limōes).

Fvitar o "surmenage" fi-icr. sexual e intelectual.

Consideram-se tambem formas frustras da insuficiencia suprarrenal certas formas de estados constitucionais astênicos, com hijmtensão ou instabilidade da pressāo arterial, com fácil fadiga fisica e intelectual.

\section{DOENÇA CELIACA OU DOENÇA DE GEE - HERTER - HEUBNER}

Esta doença näu frequente tem-se relacionado nos ultimos anos pelos auctores, a una disfuncção de suprarrenal; um argumento de valor para us que sustentam essa relação é a semelhança do quadro clinico da doença expontanea com o da doença experimental e as lesĩes anatomicas iguaes, alem da reação á terapia hormonal.

O quadro clinico da doença celiaca está caracterizado pelos seguintes simptomas:

1) diarréa, e dilatação intestinal que causa distensão abdominal ;

2) hidrolabilidade ;

3) hipofosfatemia:

4) diminuição do crescimento e osteo-porose;

5) grande hipotonia dos musculos estriados;

6) anemia por hiproglobulia e hipocromia. 
LASZT e VERSÁR reproduzem em animais este quadro, intoxicando cronicamente ratos, com acido monoiodoacético. Os simptomas são os mesmos, e na autopsia acham sempre uma hipertrofia considerarel das suprarrenaes, que para esses auctores, seria compensadora.

Eles pensam que tanto na doença expontanea como na experimental ha um mecanismo comum e que tudo seja devido a uma insuficiencia suprarrenal, ainda mais que a intoxicação monoiodo acética pode-se curar com hormonio cortical.

No animal intoxicado ha uma inhibição dos processos de fosforilização nos musculos.

\section{HIPERCORTICO — SURRENALISMO}

\section{SINDROMA GÊNITO-SUPRARRENAL (Apert)}

Congênito: pseudohermafrodismo;

Infaância: puberdade precoce.

Post- puberal: virilismo suprarrenal.

Estes quadros seriam devidos á ação do hormônio masculinizante da cortical sôbre a glandula sexual ou tambem, como afirma $\mathrm{K}_{\mathrm{RABBE}}$, parece devido a tumor suprarrenal formado por células testiculares incluidas na suprarrenal.

Pseudohermafrodismo: Mais frequente na mulher. E' um quadro caracterizado pela presença de glandulas sexuaes de um sexo, e caracteres secundarios do outro.

Genitais externos: Masculinos. Hipertrofia do clitoris, com ereção ou não. Bolsas sem testiculos. Prostata e vesiculas seminais.

Genitais internos: femininos. Ovários atróficos. Amenorréa.

Pelos com disposição masculina. Voz rouca.

Psiquismo geralmente masculino.

Anatomia patológica: Tumor benigno da suprarrenal. Raramente revelado pela radiografia.

Puberdade precoce: Geralmente antes dos 10 annos.

Desenvolvimento precoce da sexualidade: A puberdade aparece entre 6-8 anos nos meninos: desenvolvimento precoce muscular e osseo e dentario. Abundantes pelos de tipo adulto; voz grossa. Penis, testiculos e bolsas muito desenvolvidos, como no adulto.

Nas meninas: a menstruação aparece muito cedo (6 anos mais ou menos) tambem em desenvolvimento precoce osseo e dentario, pelos pubianos ${ }^{-} \mathrm{e}$ axilares como na adulta, clitoris e labios desenvolvidos.

Frequentemente se observa em ambos os sexos uma obesidade de tipo hipofisario.

Virilismo suprarrenal: No sexo feminino aparecem caracteres sexuais masculinos: regressão dos caracteres femininos.

Amenorréa quasi sempre brusca e definitiva. Perda da libido. - Hipertrofia do clitoris: atrofia dos grandes labios e utero. 
Hirsutismo: aparecem pelos na harba, bigodes, mamelão, linha branca abdominal. Pelos pubianos adquirem tipo masculino.

Voz grave; troca do caracter. Aumento da força muscular.

Aumento do metabolismo basal. Aumento do colesterol sanguineo, e dos cloretos, aumento de hormonas androgenicas na urim, aunne'utu) de $\mathrm{Na}$ (" diminuição do $\mathrm{K}$.

Policitemia- Hipertensão com bradicarclia.

Frequente obesidade, e estrias e pigmentaçōes da pelle.

Inatonia patológica: Descle uma simples hiperplasia a um arle. nona benigno, ou mais raramente um córticosurrenaloma maligno.

Fstes sindromos genito-suprarrenais podem tambe'm ser devidos a um adenoma basófilo de lobo anterior da hipofise ou un arrenoblastona in ovario. Frequente obesidade, e estrias e pigmentaçōes da pelle.

\section{HIPERSUPRARRENALISMO MEDULAR}

\section{PARAGANGLIONA HIPERTENSIVO}

Conicsa: entre os 25 a 35 anos. Afecção muito rara, descrita por DANZELOT em 1928.

Patogenia: Para VAquez, o quadro parece ser devirlo a uma adrenalinemia massiça que excitando o esplancnico parece causar uma vasoconstrição de seu território arterial.

Sintomas: Crises paroxisticas espontaneas ou depois de uma emocão, que duram de 10 a 15 minutos com: dispnéa, dores anginoides pectornis e abdominais, caimbras nas pernas. Hipertensão (22-25 de máxima), taquicardia (100-120 por minuto). Rosto pálido e angustiado, extremidades frias. Suores profusos. Náuseas, vomitos. Sensação de formigamento e caimbras nas extremiclades, cefaléa. tremor. Hiperglicemia, glicosuria. A ausscultação revela um forte clangor do $2 .^{\circ}$ tono aórtico. Uma vez parada a crise, cai a pressão e fica uma extrema fadiga. Quando as crises se repetem a meude o enfermo pode morrer de uma insuficiencia cardiaca ou pode passar á cronicidade motivando-se uma hipertensão permanente.

Anatomia: Foram achados tumores de células adrenaligenas.

Tratamento: A extirpação do tumor faz desaparecer as crises.

\section{SINDROMA SUPRARRENO-VASCULAR DE JOSUE}

Hipertensão: (Max. 20-21) no começo ha crises vasculares para logo ser permanente.

Hipertrofia cardiaca e ruido de galope: Esclerose aórtica.

Anatomia patológica: Foram constatados na autópsia; uma hiperplasia cortical e medular. 


\section{TUMORES DA SUPRARRENAL}

Os tumores que se encontram na suprarrenal podem derivar de diversos tecidos; em nosso caso particular nos interessam sómente aqueles que ocasionam transtornos de ordem endocrino e são tumores da cortical. KurzroK R. e colaboradores, classificam os tumores da suprarrenal da seguinte forma:

a) derivados do tecido conjunctivovascular, linfatico, nervoso, etc.... :

fibromas, neuro-fibromas, miomas, osteomas, lipomas, melanomas, hemangiomas, linfangiomas.

b) derivados da medular (Goldzieher): (1)

da simpatogonia;

do simpatoblasto;

do feocromoblasto;

do ganglio simpatico: ganglioneuromas;

do feocromocito: paragangliomas. (2)

c) derivados da cortical (Ewing):

hiperplasias ou nodulos de celulas corticais;

adenomas a celulas da cortical;

carcinomas. (Masson distingue: Epiteliomas trabeculares e Epiteliomas atipicos.

A adrenalina é produzida sómente por tumores formados por celulas maduras simpaticas: o feocromocitoma; este é o unico tumor medular que causa a hipertensão paroxistica (hipertensão, palpitação, dispnéa, tonturas,...). Os feocromocitonas podem observar-se em qualquer parte onde se encontre feocromocitos: na suprarrenal medular, no orgão de Zukerkandl, no corpo carotidiano, e estão dotados de atividade secretoria e de grande teór de adrenalina.

Já falamos dos simptomas causados por esses tumores no paraganglioma hipertensivo.

Os tumores que ocasionam os transtornos endocrinos são os tumores corticais, cuja caracteristica clinica é a masculinisação.

Póde aparecer em diversas idades da vida:

a) No homem antes da puberdade determina uma puberdade precoce de tipo herculeo: grande desenvolvimento muscular e sobretudo piloso (que se distribue pelo corpo todo); dentição rapida; pele grossa e seca com frequente acne; as vezes desenvolvimento precoce intelectual.

(1) Masson (Embriogenose do simpatico):

simpatogonia $\left\{\begin{array}{l}\text { linéa nervosa: simpatoblasto }\left\{\begin{array}{l}\text { cel. ganglionar } \\ \text { glia periferica }\end{array}\right. \\ \text { diferenc. gland.: cél. parasimpatica ou feocromoblasto: célula mai de }= \\ \text { cels. feocromas e paraganglios }\end{array}\right.$

(2) Da celula simpatica embrionaria nascem os neuroblastomas; da celula dif. em sentido de cel. gangl. nascem os ganglioneuremas; paragangliomas ou feocromocitomas. 
Jipo.s da pi:berdade póde deterninar uma obesidade crum hipertricose (lembrando o sindrome de Cushing).

b) $\mathrm{Na}$ mulher em idade muito jovem ou em casos congenitus: ccasiona o chamado pseudo-hermafroditismo:

Antes da pulirdade determina um virilismo com frequente obesidade. A obesidade é de preferencia facial e troncular; hijertrofia muscular: hipertricose, voz rouca; alteraçōes da pele que se torna seca, avermelhada, grossa e frequentemente com acne; do lado sexilal: pubis hipertricotico e a disposição nuasculina, labios maiores hipertrofiados assim como tambem o clitoris; parada da menstruação.

Li zida scrual: menstruaçōes escassas e depois amenorréa. falta de libido. frigidez; hipertricose, frequente cefaléa; olsesidade do tipo Cushing (as vezes com rosto congestionado), voz masculina; pele grossa e seca; hipertrofia dos grandes labios e do clitoris. Nas jovens is seios não se desenvolvem, e parecem de tipo masculino.

Frequentemente hipertensão, continua ou paroxistica, cefaléas, edemas; forte desenvolvimento muscular.

Depois da extirpação do tumor reaparece a menstruação, o libido, caem os pelos, diminue o clitoris, abaixa a pressão, etc.

Na ucnopausa: obesidade e hipertricose, sem transtornos sexuais. As vezes diabetes (no tipo Achard-Thiers).

Diagnostico: O diagnostico do tumor suprarrenal é muitas rezes dificil de ser demonstrado, devendo-se recorrer a diferentes meios para a confirmação:

raios $\mathrm{X}$, sobretudo en pielograma;

injeção intraperitoneal de ar (metodo de Langeron);

injeçāo de ar no espaço da facia perirenalis (met. Caselli);

dosagen de hormonios; R. T. FRANK (1934) demonstrou a produção de grande quantidade de foliculina, sem aumento de hormonio gonadotropo e com test negativo da gravidez.

Diagnostico diferencial: $\mathrm{O}$ diagnostico tem as vezes considerave! dificuldade; o hirsutismo ou sindrome genito suprarrenal lode observar-se em quadros clinicos de origem diversa: no sindrome de Cushing, no arrhenoblastoma do ovario, nos tumores da suprartenal (cortical), em certos tumores do timo, ficando ainda algumas tormas leves que não respondem a nenhuma causa patologica, e consideradas de causa idiopatica.

Do ponto de vista do hirsutismo puro segundo Goldzieher e Koster o hirsutismo de origem pituitaria : é de tipo penugem, sedoso e mais denso no rosto.

O de origem ovarico: é universal, de tipo masculino.

() de origem suprarrenal: constituido de pelos grossos, escuros, localisarlos na barba e nos labios e tambem em todo o corpo.

No hirsutismo pineal, geralmente ocasionado por teratomas, ha "uasi sempre puberdade precoce, sintoma de tumor cerebral, e falta a obesidade com estrias. 
No sindrome de Cushing o quadro é mais completo e póde se verificar pela radiografia o tumor hipofisario. E' necessario notar porém que foram descritos na literatura casos de sindrome de Cushing com hipofise intata achando-se contudo tumor da cortical suprarenal e até uma simples hiperplasia dela.

No arrhenoblastoma de Meyer é frequente a falta de obesidade e são ha hipertensão.

Anatomia patologica: Nos casos de adenoma suprarrenal con virilismo, as celulas, segundo os estucies de Brostner e Vines em 1933, confirmados depois por GoLDZIHER e KOSTER, apresentam graruulações fucsinofilas; mesmo nos casos de obesidade com visilismo, sem tumor da suprarrenal vêm-se essas granulações nas celulas da zona reticulada da cortical engrossada.

Nos casos de tumores sem transtornos sexuais não se notam essas granulações. Por isso estes autores relacionam as granụlações fucsinofilas com o virilismo; ha sempre tambem aumento dos lipides. As metastases destes tumores produzem os mesmos sintomas endocrinos. PoOKe e Callow em 1939 encontram grande quantidade de substancias androgenas e de trans-dehidro-androsterona no sangue em casos de sindrome de Cushing por tumor suprarrenal; R. T. FRANK achou grande quantidade de substancias estrogénicas em dois casos. Já dizemos os resultados dos estudos de Crooke. (1935) em varios casos de sindrome de Cushing estudados por ele.

Tratamento: As indicações terapeuticas são: a radioterapia e a extirpação do tumor, que pódem melhorar o quadro clinico.

\section{BIBLIOGRAFIA}

1 - Cannon W. B. - The Adrenal Medulla. Bull. of the N. Y. Acad. of Med., January, 1940.

2 - Bauer J. - Rindenhormon. Klin. Woch. 1935, s. 361.

3 - Loeb F. - Adrenal cortex. J. Amer. Med. Ass. 1935, pg. 177.

4 - Tadnea S. - Nebennieren. En: Normale und krankhafte Steuer. Adams. 1937, edição Fischer, Jena.

5 - Marañon G. - Insuficiencia suprarrenal. O Hospital. 1938, Jan.

6 - Annes Dias - Os problemas clinicos da insuficiencia suprarrenal. Acta Medica. N.o 1 - 1938.

7 - E. RYNeARson - The treatment of Addison's disease. J. A. M. A. 1938, pg. 897.

8 - Annes Dias - Insuficiencia suprarrenal. Presse Med. 6-11-1940, p. 897.

9 - Marañon - Sindromes abdominales de origen endocrino. Bol. Acad. Nac. Medic. de Buenos Aires. 10-1939.

10 - Idem. Insuficiencia suprarrenal y función sexual. Presse Méd. 1936, pg. 2057.

11 - R. Rivorre - Le traitement de la maladie d'Addison. La Pr. Mél. 1935. 13-VII, pg. 1122. 
12 - G. W. ThOR:, W. M. Firor - Desoxycorticosterone Acetate Therapy in Addison: Disease. Clinical considerations. The J. A. M. A. June 29-1940. pg. 2517.

13 - E. PERRY MCCURAciH, F. J. RIAN - The use of desoxycorticosterone acetate in Add.'s discase. Idem. Idem, pg. 2530.

17 - E. S. Gorbon - Use of desoxycort. and its esters in Add.'s disease. Idem. Idem, pg. 2549.

15 - Thомеsо: e colab. - Endocrinology. Vol. 24. VI-1939.

16 - Cahill. Lofb, Kurzrok. Stout, Smith - Adrenal Cortical Tumors. Clinical Congress of the Amer. Coll. of Surgeons. S. Francisco. October 28 - November 1-1935.

Surgery, Gynecol. and Obst. February 15-1936.

17 - Goldziener and Koster - Am. J. Surg. 1935, $77: 93$.

18 - Brostrer and Vinfs - The Adrenal Cortex. London: Lewis. 1933.

Novidades bibliograficas.

\section{ENDOCRINOLOGIA}

Compendio teorico-pratico

\section{pelo Prof. Dr. D. M. GONZALEZ TORRES}

Um volume encadernado em tela, com 350 pgs. e 53 figuras. Preço do exemplar: $60 \$ 000$

Pedidos a: LUIS DUBREZ — Rua S. Bento 357, 2. ${ }^{\circ}$ andar, S. 3.

\section{LIVROS DE MEDICINA}

Consultem preços e condições com os seus colegas:

EDGARD SCHROEDER SAN JUAN

RUA BARÃO TATUHY, 120

3. ANO

5-1608

PAULO PEREIRA CORREIA 


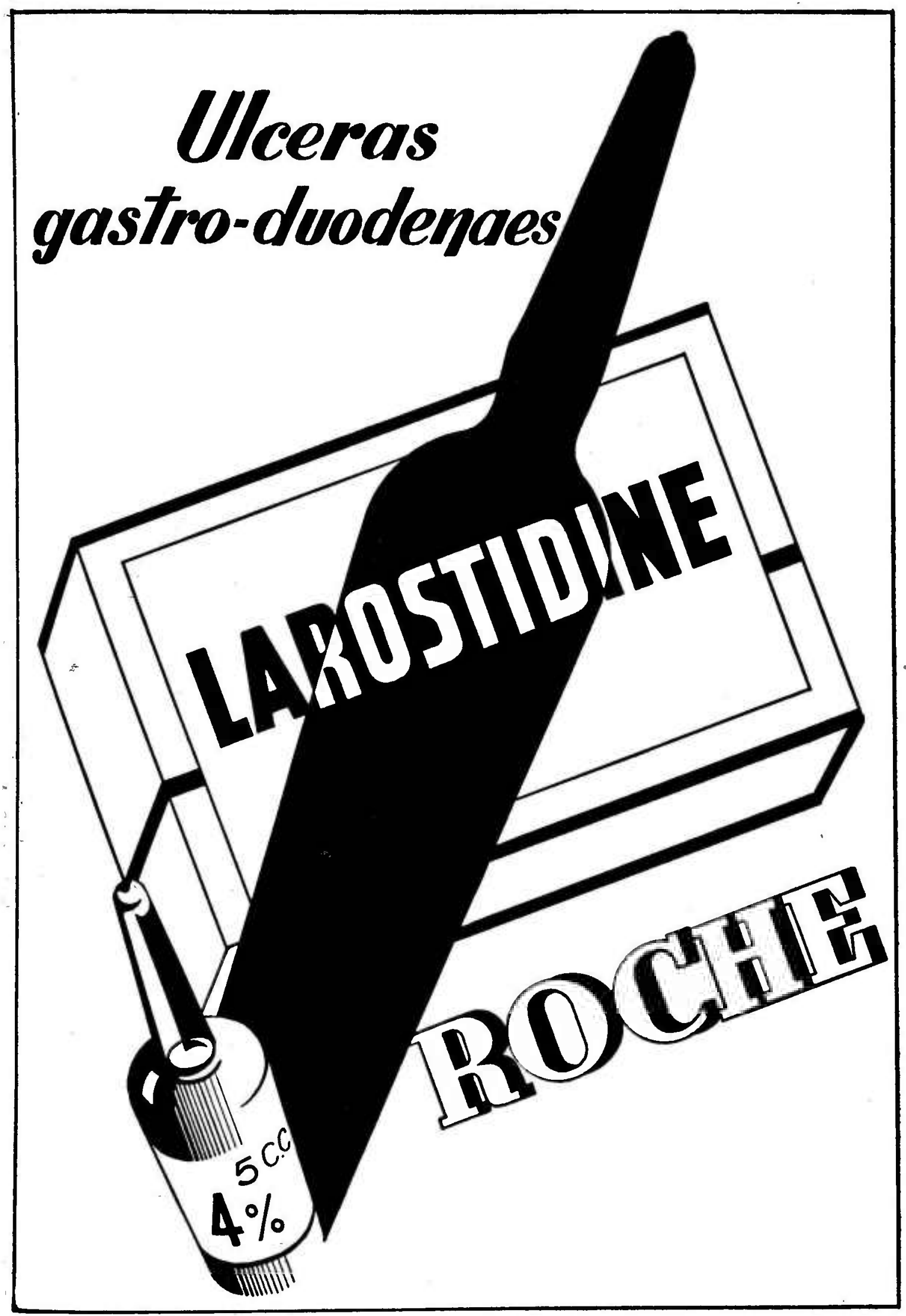

PRODUCTOS "ROCHE"S. A. - RUAEVARISTO DA VEIGA, IOI-RIO 


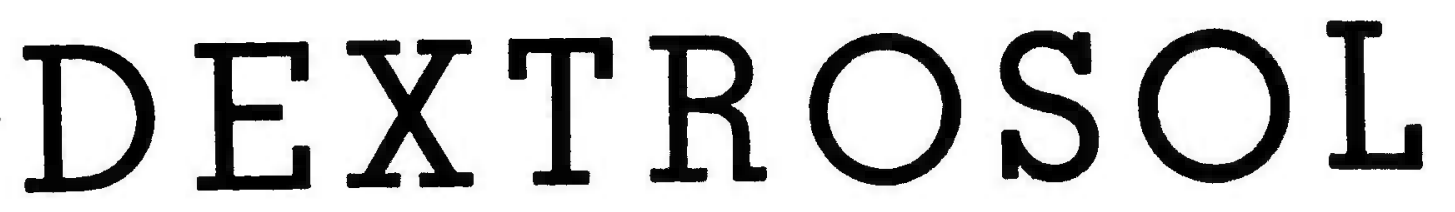

( $G L U C O S E-d)$

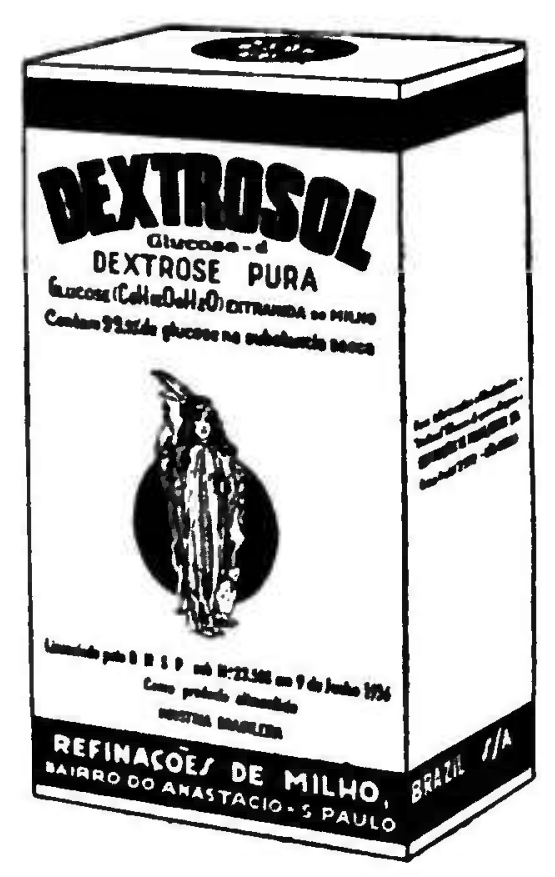

"DRENA AGUA dOS TECIDOS PARA A CIRCULAÇÁo, ELIMINANDO EDEMAS, AUMENTANDO O VOLUME SANGUINEO E PROMOVENDO A DIURESE"

E. MEYER - Usos Terapeuticos das Injeçōes Endovenosas de Soluçōes de Glucose) Zentralb. f. klin., Med. 102.343, 1925. Abst. J. A. M. A. 86.521, 1926. 